\title{
Sparse Source EEG Imaging with the Variational Garrote
}

\author{
Hansen, Sofie Therese; Stahlhut, Carsten; Hansen, Lars Kai
}

Published in:

2013 International Workshop on Pattern Recognition in Neuroimaging (PRNI)

Link to article, DOI:

10.1109/PRNI.2013.36

Publication date:

2013

Link back to DTU Orbit

Citation (APA):

Hansen, S. T., Stahlhut, C., \& Hansen, L. K. (2013). Sparse Source EEG Imaging with the Variational Garrote. In 2013 International Workshop on Pattern Recognition in Neuroimaging (PRNI) (pp. 106-109). IEEE. https://doi.org/10.1109/PRNI.2013.36

\section{General rights}

Copyright and moral rights for the publications made accessible in the public portal are retained by the authors and/or other copyright owners and it is a condition of accessing publications that users recognise and abide by the legal requirements associated with these rights.

- Users may download and print one copy of any publication from the public portal for the purpose of private study or research

- You may not further distribute the material or use it for any profit-making activity or commercial gain

- You may freely distribute the URL identifying the publication in the public portal

If you believe that this document breaches copyright please contact us providing details, and we will remove access to the work immediately and investigate your claim. 


\title{
Sparse Source EEG Imaging with the Variational Garrote
}

\author{
Sofie Therese Hansen, Carsten Stahlhut, Lars Kai Hansen \\ Department of Applied Mathematics and Computer Science \\ Technical University of Denmark \\ Kgs. Lyngby, Denmark \\ sofha@dtu.dk,csta@dtu.dk,lkai@dtu.dk
}

\begin{abstract}
EEG imaging, the estimation of the cortical source distribution from scalp electrode measurements, poses an extremely ill-posed inverse problem. Recent work by Delorme et al. (2012) supports the hypothesis that distributed source solutions are sparse. We show that direct search for sparse solutions as implemented by the Variational Garrote (Kappen, 2011) provides excellent estimates compared with other widely used schemes, is computationally attractive, and by its separation of 'where' and 'what' degrees of freedom paves the road for the introduction of genuine prior information.
\end{abstract}

Keywords-EEG; Imaging; Variational Garrote; LASSO; Sparse Bayesian Modeling; Sparsity

\section{INTRODUCTION}

We are interested in real-time imaging of human brain function by electroencephalography (EEG). The EEG imaging problem is of significant theoretical interest and realtime EEG imaging has many potential applications including quality control, in-line experimental design, brain state decoding, and neuro-feedback. In mobile applications these possibilities are attractive as elements in systems for personal state monitoring and well-being, and indeed in clinical settings where proper care requires imaging under quasinatural conditions [1]. The first real-time mobile systems are based on reconstruction methods using basic Tikhonov regularization [1]. However, the computational challenges induced by the highly ill-posed nature of the EEG imaging problem escalate in mobile real-time systems and new algorithms may be necessary [2].

In recent work by Delorme et al. [3] it is argued that independent components of EEG signals are dipolar in nature. In particular it was shown that a direct dipolar fit can explain much of the spatially distributed signal measured in scalp electrodes. This is in line with a large literature, see e.g. [4] and references herein, suggesting sparse localized sources and motivates reconstruction algorithms that emphasize sparsity, thus contrasting the distributed spatial source patterns promoted in classical alternatives [5].

Unfortunately, the quest for sparse solutions to the EEG imaging problem is combinatorial and an exact solution will not be feasible in realistic real-time systems. Many promising approximation schemes have been proposed for the general problem and many have been applied to the EEG imaging problem. We here investigate a recent alternative for sparse recovery proposed by Kappen [6]. The soft active set construction is of particular interest to real-time EEG as it enables separation of the location and magnitude estimation aspects of the reconstruction task, and furthermore leads to a relative low-complexity set of non-linear equations that are iterated towards the solution.

\section{THE EEG INVERSE PROBLEM}

In the quasi static approximation the relation between dipolar sources placed at the cortical surface $w_{i}$ and the measured potentials at multiple scalp locations $y_{\mu}$ is instantaneous and linear $y_{\mu}=\sum_{i=1}^{n} w_{i} X_{i \mu}+\xi_{\mu}$. We have denoted the forward model by $X_{i \mu}$ and allowed for measurement noise $\xi_{\mu}$, which is further assumed to be independent of the source signal. In a typical laboratory setting the number of measured scalp signals $p$ can be $32-256$, while the source distribution can be represented by $n=1000-10,000$ locations. Thus we face a severely underdetermined problem and regularization is necessary to ensure a well-defined solution, see e.g., [7] for an early review. As we have noted key processes appear to be rather dipolar, thus searching for sparse localized solutions seems well-motivated.

\section{THE VARIATIONAL GARROTE}

The so-called Variational Garrote (VG) introduces sparseness into the regression problem by adding the binary 'location' variable $s_{i} \in\{0,1\}$ for absent/present parameters [6]. Thus, the modified linear problem reads

$$
y_{\mu}=\sum_{i=1}^{n} w_{i} s_{i} X_{i \mu}+\xi_{\mu}
$$

The location variable is a latent binary variable with a prior $p(\mathbf{s} \mid \gamma)=\prod_{i=1}^{n} p\left(s_{i} \mid \gamma\right)$ where $p\left(s_{i} \mid \gamma\right)=\frac{\exp \left(\gamma s_{i}\right)}{1+\exp (\gamma)}$. Parameter $\gamma$ will in general be assumed negative $\gamma<0$, reflecting a bias towards sparsity.

The optimal solution to (1) can be obtained with a variational approximation proposed in [6]. First the posterior probability of the model given the data is established based on a Gaussian noise assumption, $\xi \sim N\left(0, \beta^{-1}\right)$, $p(\mathbf{s}, \mathbf{w}, \beta \mid \mathbf{D}, \gamma) \propto p(\mathbf{w}, \beta) p(\mathbf{s} \mid \gamma) p(\mathbf{D} \mid \mathbf{s}, \mathbf{w}, \beta)$, with $\mathbf{D}$ being the full data set, while the prior over sources and noise variance is assumed to be uniform $p(\mathbf{w}, \beta) \propto 1$. The 
discrete variable $\mathbf{s}$ is marginalized out, giving rise to the marginal posterior, $p(\mathbf{w}, \beta \mid \mathbf{D}, \gamma)$. The resulting expression to maximize is now

$$
\log p(\mathbf{w}, \beta \mid \mathbf{D}, \gamma) \propto \log \sum_{\mathbf{s}} p(\mathbf{s} \mid \gamma) p(\mathbf{D} \mid \mathbf{s}, \mathbf{w}, \beta)
$$

Invoking Jensen's inequality and a variational posterior over source locations, $q(\mathbf{s})$, we bound the log-likelihood in (2) from below by

$$
-\sum_{\mathbf{s}} q(\mathbf{s}) \log \frac{q(\mathbf{s})}{p(\mathbf{s} \mid \gamma) p(\mathbf{D} \mid \mathbf{s}, \mathbf{w}, \beta)} \equiv-F(q, \mathbf{w}, \beta) .
$$

The variational free energy $F(q, \mathbf{w}, \beta)$ is minimized, corresponding to maximizing the log-likelihood (2). We assume $q(\mathbf{s})=\prod_{i=1}^{n} q_{i}\left(s_{i}\right)$ and factors $q_{i}\left(s_{i}\right)=m_{i} s_{i}+\left(1-m_{i}\right)(1-$ $s_{i}$ ), where $m_{i}$ is the probability that $s_{i}=1$ [6].

As noted, the EEG problem is severely underdetermined, therefore we can simplify the model using a dual formulation with update rules for $p$ Lagrange multipliers $\lambda_{\mu}, \hat{y}_{\nu}$ [6]

$$
\begin{aligned}
A_{\mu \nu} & =\delta_{\mu \nu}+p^{-1} \sum_{i=1}^{n} m_{i} X_{i \mu} X_{i \nu} /\left(\left(1-m_{i}\right) \chi_{i i}\right), \\
y_{\mu} & =\sum_{\nu=1}^{p} A_{\mu \nu} \hat{y}_{\nu}, \quad \beta^{-1}=p^{-1} \sum_{\mu=1}^{p} \hat{y}_{\mu} y_{\mu}, \\
\lambda_{\mu} & =\beta \hat{y}_{\mu}, \quad w_{i}=\frac{1}{\beta p \chi_{i i}\left(1-m_{i}\right)} \sum_{\mu=1}^{p} \lambda_{\mu} X_{i \mu}, \\
m_{i}^{-1} & =1+\exp \left(-\frac{\beta p}{2} w_{i}^{2} \chi_{i i}-\gamma\right), \quad v_{i}=m_{i} w_{i} .
\end{aligned}
$$

The computational complexity is dominated by a term $\propto n p^{2}$ (4) which is much lower than the $n^{3}$ complexity of a direct implementation.

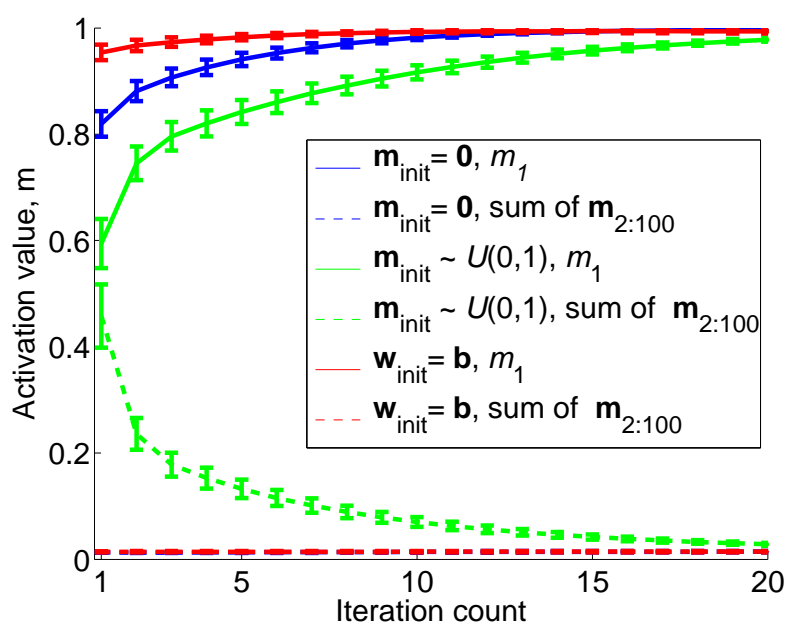

Figure 1: Simulated data $(p=50, n=100$, single active source, $\mathrm{SNR}=-1.4 \mathrm{~dB}$ ) applied to VG. Activation of the true source (full), sum of non-sources (dotted). When using $\mathbf{m}_{\text {init }}=\mathbf{0}$ the planted source is recovered in $88.3 \%$ of the 1000 repetitions, for $\mathbf{m}_{\text {init }} \sim U(0,1) 88.7 \%$ and using $\mathbf{w}_{\text {init }}=\mathbf{b} 89.6 \%$. The found sources with $m_{i}>0.5$ are considered recovered.

\section{EVALUATION}

We investigate the Variational Garrote in a series of simulation experiments, and in a benchmark EEG data set. First we investigate a simple setup based on a random forward model, while the remaining simulations and the benchmark data are based on a high-dimensional EEG Boundary Element Method (BEM) forward model [8]. For the first set of simulations we form $p=50$ measurements and $n=100$ unknown sources and apply the noise precision $\beta^{-1}=1$. Here we let a single source element in the 'true' generating model be set to unity, while the rest are set to 0 . VG is run on this data set with $\gamma=-10$ (found in pilot experiment) and $\mathbf{m}$ initialized with three different strategies. The swift convergence of the estimated probabilities of the location indicators is illustrated in Fig. 1.

Next, VG and three currently used approximate solvers; least absolute shrinkage and selection operator (LASSO) [9], Forward selection [10], and Sparse Bayesian Modeling (SBM) [11], are tested in a more realistic EEG setting using synthetic sources. The latter consists of 10 sources set to the value 1 , and the rest 0 . However, now using a normalized forward model as $\mathbf{X}$ created using OpenMEEG [8] mapping $n=8196$ sources to $p=128$ electrodes.

The four methods all have a single hyper-parameter to tune, in the VG we follow [6] and tune the sparsity parameter $\gamma$. The data set is first split into a training and test set with $p_{\text {test }}=10$ and $p_{\text {train }}=118$. Within the training set we further perform $K$-fold cross-validation to tune the four methods' hyper-parameters. We use $K=2, \ldots, 15$, i.e., the training set is subdivided to consist of a training set $((K-1) / K)$ and a validation set $(1 / K)$. For each $K$, performances are reported in terms of the normalized mean

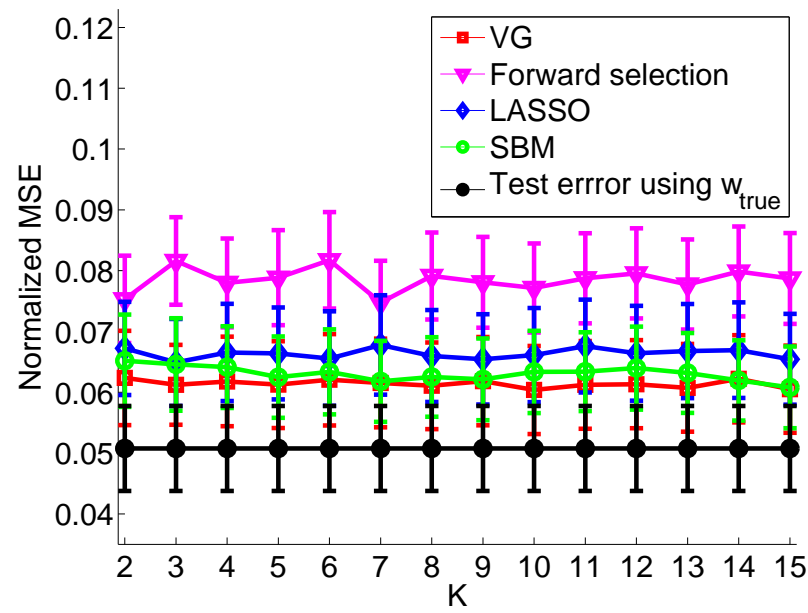

Figure 2: Normalized mean squared test error after performing 50 two-leve $K$-fold cross-validations, $K=2, \ldots, 15$. The algorithms are optimized wrt one parameter; for VG the sparsity level $\gamma$, for Forward selection the size of the active set, for LASSO the regularization parameter $\lambda$ and for SBM the precision of the noise $\beta$. The solution of $\mathrm{VG}$ is in the form of $\mathbf{v}$, while the remaining use the weight distributions directly. Ten sources out of 8196 are defined to be active in the 'true' weight distribution. 


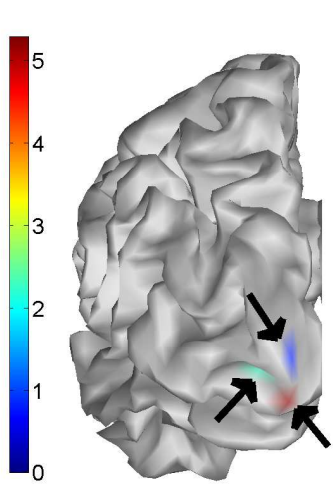

(a) VG

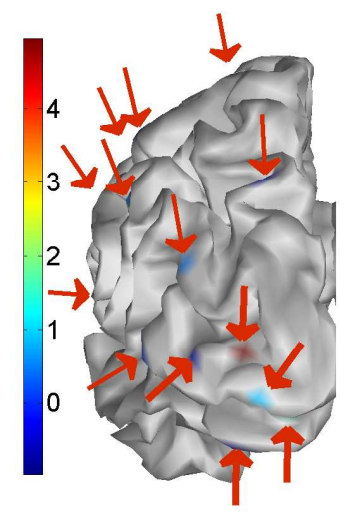

(b) Forward selection

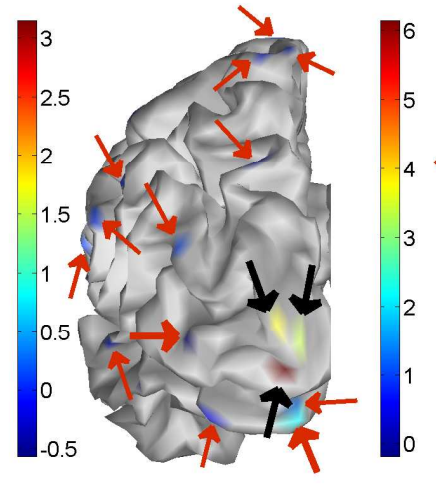

(c) LASSO

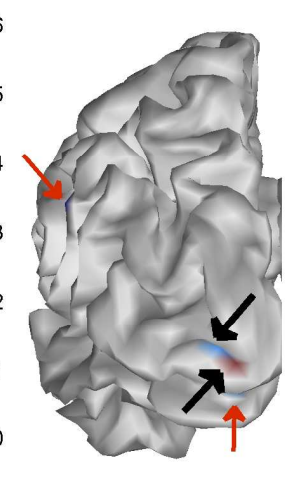

(d) SBM

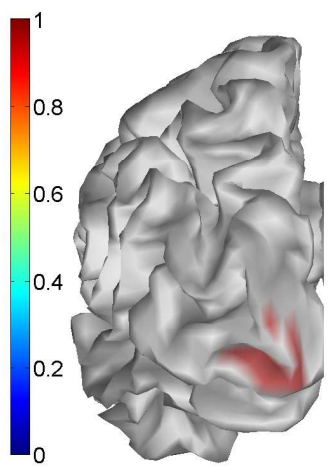

(e) True

Figure 3: Sources estimated through ten-fold cross-validation in the context of a 3D cortex structure are compared with the 'true' distribution. The solution for VG corresponds to $\mathbf{v}$ including a threshold on the activation so that, $P\left(s_{i} \mid \mathbf{D}\right)=m_{i}>0.5$ (maximum marginal posterior). The solutions presented for the three other algorithms are the weight distributions with a treshold of $10^{-10}$. Heavy or light arrows indicate sources with magnitudes larger or less than 0.5 , respectively. Black arrows indicate true sources and red false sources. View is from the back of the left hemisphere. No sources are found in the right hemisphere for VG, only low-strength sources for LASSO, one low strength for SBM while Forward selection returns many distributed sources. Note individual color maps are used.

squared error (nMSE). The above procedure is repeated 50 times. Fig. 2 compares the performances of the four methods. To set a scale we also include the nMSE for the 'true' source distribution. We find that VG outperforms the alternatives for all $K$. It is noted (not shown) that the convergence speed of VG in the applied 'EEG setting' is similar to that of the simple setup applied in Fig. 1. Interestingly, the performances of the methods with their respective optimized hyper-parameters are stable with respect to fold size $K$. Further, inspection of the estimates reveals that, e.g., LASSO - also referred to as minimum current estimate in the present context [12] - is less sparse than VG, and in fact has many small 'false' sources. Fig. 3 visualizes the spatial structure of the found sources in the context of a 3D 'cortex'.

For the simulation we also check how well VG with optimization of sparsity using the electrode cross-validation procedure is able to identify the actual source locations. For this experiment we plant 10 sources and estimate source distributions for a range of sparsity parameters $(\gamma)$. In Fig. 4 we show that the cross-validation error as function of the sparsity control parameter indeed is minimized in the same range as the source retrieval index $F_{1}=\frac{2 \cdot \text { precision.recall }}{\text { precision+recall }}$ [13] is maximized. For comparison we show the similar plot for SBM (where the cross-validated hyper-parameter is the noise precision). Here the test error-optimal solution has a somewhat lower source retrieval index than obtained by VG.

For a final test of the performance of VG in the context of 'real' EEG we turn to the SPM face recognition benchmark data [14].We focus on reconstructing the sources and their activation in a time window $100 \mathrm{~ms}<t<200 \mathrm{~ms}$, at sampling rate fs $=200 \mathrm{~Hz}$. We make a simple extension of the model to allow for a time constant $s_{i}$ with time varying activation strengths $w_{i}$, effectively decoupling the 'where' $(s)$ and 'what' $(w)$ degrees of freedom which leads to only minor modifications to the inference scheme [15]. In Fig. 5 we show the resulting activation time courses and in the cortex inset arrows indicate the locations of the corresponding sources. Both time courses (N170 components) and locations are consistent with the general findings of [14] and [16].

\section{DISCUSSION AND CONCLUSION}

EEG imaging is a hard, underdetermined inverse problem. We hypothesize that solutions of interest are sparse and note that sparsity constraints can regularize the problem. We have shown that direct search for sparse solutions as implemented by Kappen's Variational Garrote [6] can outperform solutions based on convex relaxations (LASSO, minimum current estimate), forward feature selection, sparse Bayesian learning, both in terms of cross-validation error on test data, and in terms of quality of the solutions. In a quasi-realistic setting with an EEG forward model we found that the VG solution provides an excellent reconstruction of the planted sources. Finally, we noted that the VG model allows separation of where and what degrees of freedom, and used this to analyze a benchmark face recognition data set assuming that the locations were constant, while activations change in time. The resulting time courses and locations for a single trial were found to be consistent with the solutions proposed earlier based on averaging over multiple epochs.

\section{ACKNOWLEDGMENT}

This work is supported by the Danish Lundbeck Foundation via the Center for Integrated Molecular Brain Imaging.

\section{REFERENCES}

[1] A. Stopczynski, J. Larsen, C. Stahlhut, M. Petersen, and L. Hansen, "A smartphone interface for a wireless eeg headset with real-time $3 \mathrm{~d}$ reconstruction," Affective Computing and Intelligent Interaction, pp. 317-318, 2011. 


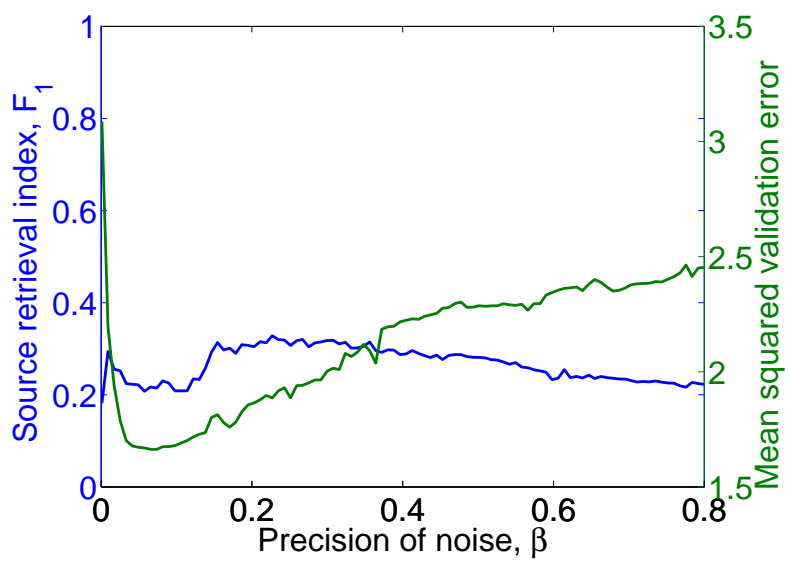

(a) Optimization comparison for SBM.

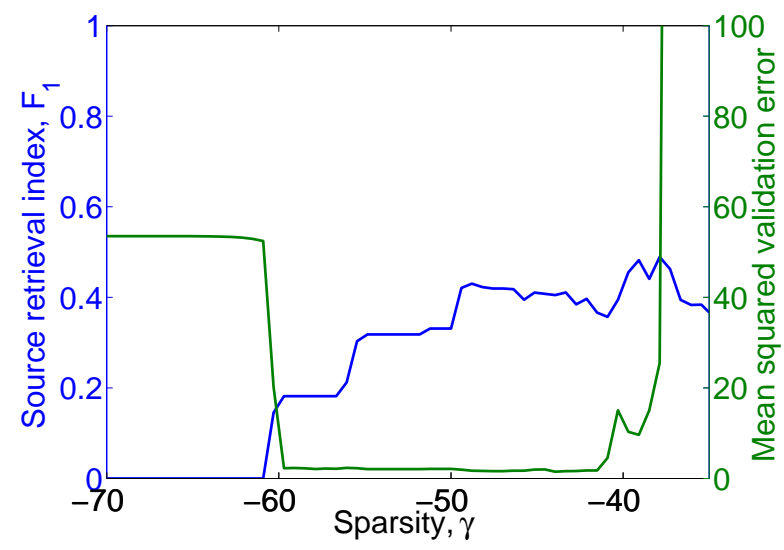

(b) Optimization comparison for VG.

Figure 4: Comparison of ability to recover sources and obtained validation error. A synthetic source distribution with ten planted sources is applied together with an EEG forward model as input, mapping $n=8196$ sources to $p=128$ electrodes. The means of ten repetitions are shown.

[2] M. Petersen, C. Stahlhut, A. Stopczynski, J. Larsen, and L. Hansen, "Smartphones get emotional: mind reading images and reconstructing the neural sources," Affective Computing and Intelligent Interaction, pp. 578-587, 2011.

[3] A. Delorme, J. Palmer, J. Onton, R. Oostenveld, and S. Makeig, "Independent eeg sources are dipolar," PloS one, vol. 7, no. 2, p. e30135, 2012.

[4] A. Gramfort, M. Kowalski, and M. Hämäläinen, "Mixed-norm estimates for the m/eeg inverse problem using accelerated gradient methods." Physics in Medicine and Biology, vol. 57, no. 7, pp. 1937-1961, 2012.

[5] R. Pascual-Marqui, M. Esslen, K. Kochi, D. Lehmann et al., "Functional imaging with low-resolution brain electromagnetic tomography (loreta): a review," Methods and findings in experimental and clinical pharmacology, vol. 24, no. suppl C, pp. 91-95, 2002.

[6] H. J. Kappen, "The Variational Garrote," arXiv preprint arXiv:1109.0486, 2011. [Online]. Available: http://arxiv.org/ abs/1109.0486

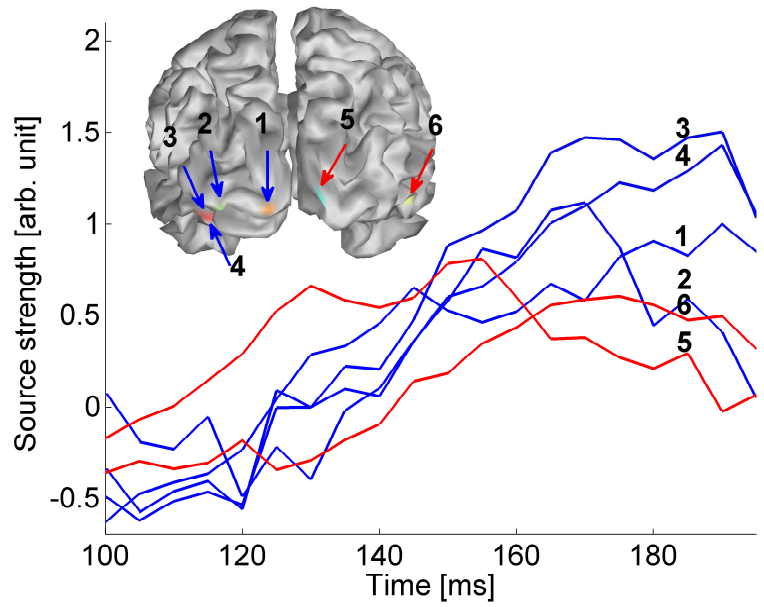

Figure 5: The sources found in a single face-evoked epoch in the time window $100-200 \mathrm{~ms}$ by the temporally extended VG. Inset shows posterior view of the cerebrum cortex with projected sources found at $180 \mathrm{~ms}$. Additionally two anterior frontal sources are found in the left hemisphere. The temporal source distribution is found using five-fold cross-validation.

[7] D. Titterington, "Common structure of smoothing techniques in statistics," International Statistical Review/Revue Internationale de Statistique, pp. 141-170, 1985.

[8] A. Gramfort, T. Papadopoulo, E. Olivi, M. Clerc et al., "Openmeeg: opensource software for quasistatic bioelectromagnetics," Biomed. Eng. Online, vol. 9, no. 1, p. 45, 2010.

[9] R. Tibshirani, "Regression shrinkage and selection via the lasso," Journal of the Royal Statistical Society. Series B (Methodological), pp. 267-288, 1996.

[10] N. Draper and H. Smith, "Applied regression analysis. Series in probability and mathematical statistics," Wiley, 1981.

[11] M. E. Tipping, "Sparse bayesian learning and the relevance vector machine," The Journal of Machine Learning Research, vol. 1, pp. 211-244, 2001

[12] K. Matsuura and Y. Okabe, "Selective minimum-norm solution of the biomagnetic inverse problem," Biomedical Engineering, IEEE Transactions on, vol. 42, no. 6, pp. 608-615, 1995.

[13] J. Makhoul, F. Kubala, R. Schwartz, and R. Weischedel, "Performance measures for information extraction," in Proceedings of DARPA Broadcast News Workshop, 1999, pp. 249-252.

[14] R. N. Henson, Y. Goshen-Gottstein, T. Ganel, L. J. Otten et al., "Electrophysiological and haemodynamic correlates of face perception, recognition and priming," Cerebral cortex, vol. 13, no. 7, pp. 793-805, 2003.

[15] S. T. Hansen, "Sparse EEG imaging," Master thesis, Technical University of Denmark, 2013.

[16] K. J. Friston, L. Harrison, S. J. Kiebel, C. Phillips et al., "Multiple sparse priors for the m/eeg inverse problem." $\mathrm{Neu}$ roImage, vol. 39, no. 3, pp. 1104-1120, 2008. 